important in compensation cases. The cause is probably a considerable indirect exposure.

\section{THE LATENCY PERIOD OF MESOTHELIOMA AMONG A COHORT OF BRITISH ASBESTOS WORKERS (1978-2005)}

G F Frost, Harding. Health \& Safety Laboratory, Buxton, United Kingdom

\subsection{6/oemed-2013-101717.273}

Objectives To investigate the hypothesis of an inverse relationship between intensity of asbestos exposure and mesothelioma latency among a cohort of asbestos workers in Britain.

Methods The Great Britain (GB) Asbestos Survey recruited asbestos workers attending medical examinations as required under the asbestos regulations in GB. Job details and smoking information were updated at any subsequent examinations. The primary outcome for this analysis was the time from first occupational exposure to asbestos to death with mesothelioma. Univariable and multivariable generalised gamma accelerated failuretime (AFT) models were used to estimate time ratios (TRs). Those who died with mesothelioma between 1978 and 2005 were included in the analysis.

Results After exclusions for missing data, there were 614 workers in the GB Asbestos Survey who died with mesothelioma between 1978 and 2005. Total follow-up time was 9,280 person-years, with a median latency of 22.8 years [ $95 \%$ confidence interval (CI) 16.0-27.2 years]. In the final multivariable AFT model, mesothelioma latency was around 26\% longer for females compared to males ( $\mathrm{TR}=1.26,95 \% \mathrm{CI}=1.15-1.37$ ), and $5 \%$ shorter for those who died with asbestosis compared to those who did not $(\mathrm{TR}=0.95,95 \% \mathrm{CI}=0.91-0.99)$ after adjustment for year of first exposure, age at first exposure, and duration of exposure. There was no evidence of associations between mesothelioma latency and occupation, smoking status and mesothelioma type in either univariable or multivariable analyses.

Conclusions The associations between mesothelioma latency and both sex and presence of asbestosis were in the directions expected if the intensity hypothesis was true. However, there was no evidence that asbestos insulation workers, who tended to experience greater asbestos exposures than other occupations, had shorter latencies than other asbestos workers. Therefore this study did not find sufficient evidence to either support or refute the intensity hypothesis. (C) Crown Copyright (2012).

\section{BIOMARKERS FOR PLEURAL MESOTHELIOMA IN THE MEXICAN INSTITUTE SOCIAL SECURITY}

${ }^{1}$ G A M Aguilar-Madrid, ${ }^{2} J u a r e z-P e r e z,{ }^{3}$ Calderón Aranda, ${ }^{2}$ Kelly García, ${ }^{1}$ Alvarado Cabrero, ${ }^{2}$ Ochoa Vázquez. 'Instituto Mexicano Del Seguro Social, Mexico, Distrito Federal, Mexico; ${ }^{2}$ Instituto Mexicano Del Seguro Social, Mexico, Distrito Federal, Mexico; ${ }^{3}$ Centro de Investigación y Estudios Avanzados. CINVESTAV. Depto Toxicología, México, $D F$, Mexico

\subsection{6/oemed-2013-101717.274}

Objective Developing a new diagnostic strategy for Malignant Pleural Mesothelioma measuring soluble proteins in plasma and pleural fluid using ELISA and qRT-PCR.

Methods Case-control study, in which the cases were incident patients with malignant pleural mesothelioma in two hospitals from Mexican Social Security (Neumology and Oncology) and there were 4 controls for each case. A questionary was applied for asbestos exposure. ELISA with double antibody was used to determine the presence of thrombomodulin and mesothelin, using known concentration curves for each one. An statistical descriptive analysis was made as well as logistic regression models and ROC curves.

Results In this study were enrolled 35 cases and 136 controls. The mean concentrations, (SD) and [min-max] in plasma for thrombomodulin were: 10.2 (7) $\mathrm{ng} / \mathrm{mL}$ [1.6-32.2] for the cases, and $5.4(5.1) \mathrm{ng} / \mathrm{mL}$ [0.78-32.5] for the controls. For mesothelin values were: $104.3(78.2) \mathrm{ng} / \mathrm{mL}$, [12.2-351.2] for the cases, and $25.2(3.2) \mathrm{ng} / \mathrm{mL},[6.5-330.3$ ] for the controls. Both biomarkers were statistically significant. In the ROC curve for both biomarkers sensibility of $82.3 \%$ and specificity of $95.5 \%$ were achieved. In the nonconditional logistic regression model, mesothelin and thrombomodulin had an OR $=1.03$ (CI: 1.02-1.05) and $\mathrm{OR}=1.08$ (CI: 1.01-1.16), respectively, adjusted for age and sex.

Conclusions A unique parameter to evaluate a diagnostic test in every situation doesn't exist, but following Sackett and Álvarez-Cáceres guidelines, our two biomarkers have a high specificity $(95.5 \%)$ and sensibility $(82.35 \%)$ with a cut-off point, in this sample, $\geq 83 \mathrm{ng} / \mathrm{mL}$ for mesothelin and $\geq 10.5$ $\mathrm{ng} / \mathrm{mL}$. Nevertheless we consider that our sample is small and that we also need to control the factors, such as diabetes mellitus, hypercholesterolemia and renal failure, that have influence in the level of both biomarkers.

\section{OCCUPATION AND MESOTHELIOMA IN PLEURA AND PERITONEUM IN SWEDEN - UPDATED INCIDENCE FOR MALES AND FEMALES 1961-2009}

${ }^{1} \mathrm{~N}$ P Plato, 'Weiderpass, 'Sparren, ${ }^{2}$ Martinsen. ${ }^{1}$ Karolinska Institutet, Stockholm, Sweden; ${ }^{2}$ Cancer Registry of Norway, Oslo, Norway

\subsection{6/oemed-2013-101717.275}

Objectives Mesothelioma incidence increased in Sweden between years 1970 and 2000, and seems to have reached a plateau in the last decade. We used data from the Swedish component of the Nordic Occupational Cancer Study - NOCCA - to study potential occupational exposures other than asbestos that may be associated with mesothelioma occurrence in men and women.

Methods Follow up was done by linkages of the Swedish NOCCA study database, including 53 occupational categories, with the population based Swedish registries (cancer, mortality, total population) from years 1961 to 2009, and matched with the Swedish version of NOCCA-JEM, which include 25 carcinogenic substances with exposure levels for 283 occupations in different employment periods from year 1945 to1994. Multivariate analysis with 20 years delay for cumulative exposure were done. Results A total of 4180 incident mesothelioma of the peritoneum and pleura were reported to the Swedish Cancer Registry (1961 2009): $26.6 \%$ were women. $83.9 \%$ were located in the pleura (18\% women). We found a significant over risk in mesothelioma in 16 of 53 occupational categories, and a clear gender difference. We observed increased risk of mesothelioma among female textile workers, building cleaners, seamstresses, printers, packers and postal workers. Men had over risks in construction workers, electricians, masons, and insulation workers; the greatest excess risk of pleural mesothelioma was observed among plumbers (SIR 5.1, 95\% CI 4.30 to 6.01 ). In multivariate analysis, controlling for other occupational exposures, significant associations were observed for asbestos exposure and mesothelioma risk.

Conclusion Exposure to asbestos is associated with incidence of mesothelioma in Sweden. There were some occupations 
however, such as for female textile workers and cleaners, where we observed an increased risk of mesothelioma without evidence of exposure to asbestos.

\section{Session: 10. Hazard identification II}

\section{OCCUPATION, INDUSTRY, AND THE RISK OF PROSTATE CANCER: A CASE-CONTROL STUDY IN MONTRÉAL, CANADA}

1J F Sauve, 'Lavoué, ${ }^{2}$ Parent. ' University of Montreal, Montreal, Canada; ${ }^{2}$ INRS-Institut Armand Frappier, Universite du Québec, Laval, Canada

\subsection{6/oemed-2013-101717.276}

Introduction Prostate cancer $(\mathrm{PCa})$ is the most common cancer among Canadian men. Age, a family history of PCa and ancestry are the only recognised risk factors, but a role for environmental influences is suspected.

Objective To explore the relationship between occupational and industry titles, and PCa risk.

Methods PROtEuS (Prostate cancer \& Environment Study) is a case-control study of PCa in Montréal, Canada including some 2000 cases and 2000 population controls aged 40-75 years. Detailed occupational histories were elicited through in-person interviews. Unconditional logistic regression was used to estimate odds ratios (OR) and 95\% confidence intervals (CI) for the association between $\mathrm{PCa}$ and employment in 98 occupations and 74 industries. Models were adjusted for age, ancestry, family history of PCa, PCa screening, income and physical activity.

Results Elevated PCa risks were found for social sciences workers (OR 1.9; 95\% CI 1.1-3.4) and metal product fabricators (OR 3.4; 95\%CI 1.3-9.3) employed $\geq 10$ years, and for nonconstruction painters (OR 3.4; 95\% CI 1.3-9.0) and plumbers and steamfitters (OR 2.4; 95\%CI 1.1-5.4) employed $<10$ years. Reduced risks were observed for farmers (OR 0.6; 95\%CI 0.40.9 ) and food services workers (OR 0.6 ; 95\% CI 0.4-0.9) employed $<10$ years, and for physical scientists employed $\geq 10$ years (OR 0.6; 95\% CI 0.5-0.9). Workers in public service, wood products, and jewellery industries had excess PCa risks. Those in agriculture, miscellaneous food, accommodation and food, air transport, and retail trade industries had reduced risks. Analyses including all subjects, or stratified according to white or blue collar status generated consistent findings.

Conclusions Our findings suggest excess PCa risks in some metal-related occupations, as well as in white-collar occupations such as in public service and social sciences. Farmers and food services workers had reduced risks. Specific occupational exposures possibly underlying these associations will be explored.

\section{THRESHOLD VALUE ESTIMATION FOR RESPIRABLE QUARTZ DUST EXPOSURE AND SILICOSIS INCIDENCE IN THE GERMAN PORCELAIN WORKER COHORT}

${ }^{1} \mathrm{P}$ Morfeld, ${ }^{2}$ Mundt, ${ }^{3}$ Taeger, ${ }^{4}$ Guldner, ${ }^{4}$ Steinig, ${ }^{5}$ Miller. ${ }^{1}$ Institut für Epidemiologie und Risikobewertung in der Arbeitswelt, Essen, Germany; ${ }^{2}$ Environ International Corporation, Amherst, Massachusetts, United States of America; ${ }^{3}$ Institute for Prevention and Occupational Medicine of the DGUV (IPA), Bochum, Germany; ${ }^{4}$ VerwaltungsBerufsgenossenschaft, Würzburg, Germany; 5 Institute of Occupational Medicine, Edinburgh, United Kingdom

10.1136/oemed-2013-101717.277

Objectives To estimate a threshold value tau for the respirable quartz dust concentration and silicosis incidence (1/1, ILO 1980/
2000) in the German porcelain worker cohort (Birk et al 2009, 2010, Mundt et al 2011).

Methods 17,144 porcelain workers $(128,688$ person-yrs) were followed for silicosis incidence (40 cases). Respirable quartz dust exposure was determined by combining detailed individual employment histories with a job exposure matrix based on 8,000 historical industrial hygiene measurements. Cox regression with age as the time variable was used to evaluate silicosis morbidity by $\log$ (cumulative quartz dust exposure $+0.01 \mathrm{mg} /$ $\mathrm{m}^{3}$-yrs) time-dependently, controlling for sex and smoking status. Curvature of the relationship was explored by restricted cubic splines (RCS, $\leq 7$ knots) and fractional polynomials (FP, degrees $\leq 5$ ). Estimation of tau was performed by subtracting a series of candidate values from the annual concentration data (setting the result to zero if negative) and recalculation of the time-dependent cumulative exposures. The partial likelihood profile was used to derive point and 95\%-confidence interval (CI) estimates. Non-nested models were compared by information criteria (AIC). Lagging exposures by 10 years, using different offsets in the log-function $\left(0,0.1,1 \mathrm{mg} / \mathrm{m}^{3}\right.$-yrs $)$ and varying sets of covariates were explored.

Results We estimated tau $=0.25 \mathrm{mg} / \mathrm{m}^{3}\left(0.95-\mathrm{CI}: 0.15 \mathrm{mg} / \mathrm{m}^{3}\right.$, $0.30 \mathrm{mg} / \mathrm{m}^{3}$ ). Applying this estimated concentration threshold led to lower degree optimal FPs and returned pronounced better fits ( AIC > 5) in log-linear Cox models, 5-knots RCS Cox models and 2-degree FP Cox models. The overall exposure-response could be appropriately described by a Cox model on log (unlagged cumulative exposure $+0.01 \mathrm{mg} / \mathrm{m}^{3}$-yrs) after applying tau $=0.25 \mathrm{mg} / \mathrm{m}^{3}$.

Conclusions A threshold Cox model fitted the data significantly better than a non-threshold model and summarised the cohort information without a loss in extracted information and much more simply than the curvilinear procedures (RCS, FP).

\section{MORTALITY PATTERNS IN A COHORT OF 70,000 WORKERS WITH BLOOD LEAD MEASUREMENTS}

${ }^{1} \mathrm{~K}$ Steenland, ${ }^{2}$ Chowdhury. ${ }^{1}$ Atlanta, United States of America; ${ }^{2}$ Emory University, Atlanta, United States of America

\subsection{6/oemed-2013-101717.278}

Objectives To determine whether adult lead exposure is associated with cause-specific mortality in a cohort with measured blood leads

Methods We studied 70,000 US adults from 11 states with blood lead levels measured between 1980-2005. Most were exposed occupationally. One-third had a single blood lead; the remainder had a median of 3 blood leads. Subjects were divided into four groups by highest blood lead $(0-5,6-24,25-39,40+$ $\mathrm{mg} / \mathrm{dl} ; 16 \%, 33 \%, 34 \%$, and $17 \%$ respectively.

Results Analyses were restricted to males (96\% of deaths). There was a strong healthy worker effect in this young cohort (SMR all causes $0.73,3561$ deaths, US referent). Most cause-specific SMRs were unremarkable. The male lung cancer SMR showed a trend of increase across lead categories (SMRs 0.49, 0.61, 0.80, $1.21,394$ deaths, test for trend $\mathrm{p}=0.003$ ). With the lowest lead category as referent in Poisson regression, lung RRs were $1.00,1.27,1.80$, and 2.85 by increasing lead category (test for trend $\mathrm{p}=<0.0001)$. Other smoking-related causes of death did not show consistent trends (eg., male COPD male SRRs 1.00, 0.21, 0.44, $0.63(\mathrm{n}=129)$, male esophageal cancer SRRs 1.00, $1.29,0.98,1.43(\mathrm{n}=40)$. There was a marked excess of ALS in 